\title{
The living realm depicted by the fractal geometry
}

\author{
Gabriele A. Losa*
}

Institute of Scientific Interdisciplinary Studies, 6600 Locarno-Switzerland

\section{Fractal geometry into the life sciences}

Most cell tissues, biological elements, physiopathologic processes and natural objects fashioned through irregular scale-free traits and spatial/temporal self-similar properties are investigable by the Fractal Geometry of B. Mandelbrot [1]. An objective unattainable with the classical Euclidean geometry developed to trace regular ideal geometrical forms practically unknown in nature. The cultural-scientific paradigm emerging from Mandelbrot's masterpiece - The Fractal Geometry of Nature - may recall the fascinating view of Leonardo da Vinci [2], who in his Trattato della Pittura [Traitise on Painting] alleged that living organisms and complex shaped structures must be tackled by $<$ figurare e descrivere >, i.e. < picture and depict > in other words by drawing and describing them as precise and realistic as possible. The basic concepts and theoretical principles of Fractal Geometry, notably "Statistical Self-Similarity and Fractional Dimension" were spread in precedented single publications followed by the first coherent essay in the early second half of the twentieth century [3]. It may be worth considering exactly how and when the "heuristic introduction" of such an innovative discipline occurred or, more pregnantly stated, as when "the irruption of fractal geometry" into the life sciences such as biology and medicine actually took place [4]. Although there is no precise date, it is generally agreed that such as introduction occurred within the "golden age" of cell biology situated between the 1960s and 1990s.

\section{The morphofunctional complexity of cells and tissues}

From the direct observation of Nature, it emerges that most cells, tissues, organs - in either the animal or vegetal worlds - are systems in which the component parts and unit fragments assemble with different levels of complexity and recursive organization at several orders of magnitude. This means that a single fragment or element may, on various scales, reproduce the whole object from which it is derived; in other words, it is self-similar, albeit in a statistical sense. With regard to the abovementioned period there was a pressing need to consider the morphological complexity of cells and tissues by using a systemic approach, whilst at the same time developing instruments that could enable the accomplishment of that goal without introducing any shape approximation or smoothing, a condition which could not be satisfactorily achieved with conventional analytical methods. In fact, the latter situation which relied on conventional disciplines such as morphometry and stereology yielded experimental data relating to the quantitative description of membranes that was usually controversial. This left many questions unresolved and thereby prevented a true consensus being reached among the investigators [5]. To highlight the striking debate that led to such turmoil within the biologist community, it might suffice to evoke the case study conducted several years earlier that related to the application of fractal geometry in cell biology; notably, the discovery that cellular membrane systems have fractal properties arose from the uncertainty of observations regarding the extent of such membranes. In fact, studies on the morphometry of liver cell membranes conducted by various laboratories reported values significant discrepant and therefore not comparable [6]. There followed much debate as to which of these estimates was correct, and whether liver cells contained 6 or $11 \mathrm{~m}^{2}$ of membranes per $\mathrm{cm}^{3}$. The question was also raised as to whether the stereological methods used were reliable, since it appeared possible that the same method might yield different results if the measurements were made under different magnifications of the electron micrographs. In fact, the systematic measurement of liver cell membranes revealed that the estimates of surface density increased with increased resolution. Shortly after the conclusion of the experimental phase, Mandelbrot suggested that the results should be interpreted with the likely effect of the "resolution scale," in analogy with the "Coast of Britain effect" [7]. The estimate discrepancy was resolved allowing to explain why measurements of irregular liver cell membranes at higher magnification yielded higher values than were obtained at lower magnification [8]. It must be stressed here that the scaling effect applies mainly to cellular membranes with a folded surface or an indented wrinkled profile, such as the inner mitochondrial membrane. In contrast, measurement of the surface density of the outer mitochondrial membrane, which is almost smooth, was only slightly affected by the resolution effect. A contemporaneous study, which investigated the gas exchange surface of the lung, revealed that the alveolar surface area was increased at increasing magnification. The inner lung surface consists of a hierarchy of successive structures, namely the alveoli, then capillaries in the alveolar wall, and finally alveolar epithelial cells with wrinkled membranes: these different structures can, however, hardly be considered as self-similar structures of a unique type, since each has its own generator, is determined by its own constructive algorithm and hence entails a different self-similar level [9]. Several authors further addressed the problem relating boundary length to resolution, namely with regard to specimens showing "non-ideally fractal" dimensions such as lung tissues but also to cell tissues in different physiopathological conditions [10-14].

\section{The quantitative description of complex shapes}

Founded upon a body of well-defined laws and coherent principles, including those derived from chaos theory, the Fractal Geometry allows the recognition and the quantitative description of complex shapes, living forms, biologic tissues, organized patterns of morphologic features correlated through a broad network of functional interactions and metabolic processes that shapes the adaptive response and makes the accomplishment of life possible [15]. Obviously, this

Correspondence to: Gabriele A. Losa, Institute of Scientific Interdisciplinary Studies, 6600 Locarno-Switzerland; E-mail: gabriele.losa@bluewin.ch

Received: April 29, 2015; Accepted: May 20, 2015; Published: May 24, 2015 
came in opposition to the ancient vision based on the rules of the Euclidean geometry and widely adopted concepts, such as homeostasis, linearity, smoothness and thermodynamic reversibility, which emanate from a cultural view but ideal of envisaging things and facts. Later on it has become evident that biologic elements, unlike deterministic mathematical structures, express statistical self-similar patterns and fractal properties within a defined interval of scales, called "scaling window ", in which the relationship between the observation scale and the measured size or length of the object can be established and defined as the fractal dimension [FD] [16]. The fractal dimension FD of a biological component remains constant within the scaling window and serves to quantify the variation in length, area or volume with changes in the size of the measuring scale. The recourse to the principles of fractal geometry has enabled to reveal that most biological elements, either at cellular, tissue or organic level, have self-similar structures within a defined scaling domain which can be characterized by means of the fractal dimension FD. However, concrete "fractality" exists only when the experimental scaling range accounts for at least two orders of magnitude, namely spans two decades on the scaling axis. Data spanning several decades of scale has been previously reported in many other fields: thus, defining a "scaling range" appears as an inescapable requisite for assessing the fractality of every biological element. Its occurrence will hence exclude any ambiguous assignment and so far strengthen the statement of Mandelbrot, $<$ fractals are not a panacea; they are not everywhere $>$. In the Epilog of his work -The path to fractals- he wrote $<$ The reader knows well that the probability distribution of fractals is hyperbolic, and that the study of fractals is rife with other power law relationships $>$. Nowadays, the Fractal Geometry provides the theoretical and methodological framework for unravel temporal processes and complex biological structures, as underlined by the increased frequency of outstanding publications, what definitely reinforces a valiant sentence written more than twenty years ago $<$ The fractal Geometry: a Design Principle for Living Organisms $>$ [17]. So far, it is worthy to point out that the fractal dimension is a statistical measure which correlates the morphological structural complexity of cellular components and biological tissues. It is also a numerical descriptor which serves to measure qualitative morphological traits and self-similar properties at various levels, cellular, tissue or organic. Mandelbrot stated in his book that $<$ A fractal set is a set in metric space for which the Hausdorff-Besicovitch dimension $\mathrm{D}$ is greater than the topological dimension DT $>$. In Nature, a fractal object is defined by its structural properties, namely by surface rugosity and irregularity or absence of smoothness, form invariance or self-similarity, and complexity. The Richardson-Mandelbrot equation provides the mathematical basis for understanding geometric and spatial fractal structures, and for measuring and interpreting them, namely

$$
\mathrm{L}(\varepsilon)=\mathrm{N}(\varepsilon) .(\varepsilon)
$$

where $L(\varepsilon)$ represents the contour length (e.g. the perimeter) of the biological component under investigation, $(\varepsilon)$ the unit length of measure and $\mathrm{N}(\varepsilon)$ the number of unit lengths $(\varepsilon)$ needed to cover the contour $\mathrm{L}(\varepsilon)$. By substituting $\mathrm{N}(\varepsilon)$ with $\left[\mathrm{lo}^{\mathrm{D}} \varepsilon^{-\mathrm{D}}\right]$ into (1), the above equation can be transformed by logarithmic procedure and rewritten as

$$
\log [\mathrm{L}(\varepsilon) / \mathrm{lo}]=(1-\mathrm{D}) \log [\varepsilon / \mathrm{lo}]
$$

which is the equation of a straight line with slope 1-D, from which the dimensional exponent $\mathrm{D}$ can be calculated to yield the numerical value of the fractal dimension FD. As mentioned in the Introduction, biological fractals also called asymptotic natural fractals show auto-similar scaling properties (fractality) within a fractal window, graphically represented by the region II in the middle of three typical

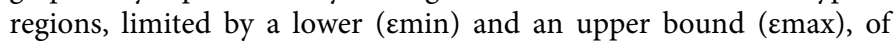
an bi-asymptotic fractal, where a straight line can be drawn and the fractal dimension [FD] calculated from its slope. While the practical evaluation of the fractal dimension could be obtained by various quantitative approaches, the box counting method easily based on counting of the non empty boxes $\mathrm{N}$ at a variable grid length $(\varepsilon)$, is by far the most reliable.

\section{Cell membranes and cell organelles}

Particularly at the electron microscopy level, the fractal analysis proved useful for an objective investigation of cell shape and cell phenotype, the fine cytoplasmic structures and the organization of cell membranes and nuclear components and other subcellular organelles, either in normal or pathological tissues and in cell cultures during time $[15,16,18]$. The fractal tool has also been employed to document the feasibility of using ultrastructural changes in cell surface and nuclear inter(eu)chromatin to assess the early phases of apoptotic cell death. Ultrastructural changes which involved a loss in heterochromatin irregularity due to its increased condensation (lower FD), were evident well before the detection of conventional cell markers, which were only measurable during the active phases of apoptosis. Furthermore, the nuclear complexity of human healthy lymphocytes was shown to undergo a reduction of FD during the apoptotic process [19]. Measuring the FD of euchromatin and heterochromatin nuclear domains helped to discriminate lymphoid cells found in Mycosis fungoides from those in chronic dermatitis [20].

\section{Membrane irregularities in hematological malignancies}

The first application of fractal morphometry to nonsolid cancers came at early last decade of nine hundred century, when human leukemia cells of lymphoid and/or myeloid origin were characterized on electron microscopic images through quantitative measurement of membrane surface properties that could be correlated with specific phenotype markers [21]. Cells isolated ex vivo from the blood of humans with acute T-lymphoid leukemia revealed pericellular membranes with a nearly smooth outline as documented by fractal dimension values significantly lower than those found for pericellular membranes of healthy blood cells. Healthy lymphocytes of B-cell lineage had a fractal dimension FD (1.20) significantly different from that of lymphocytes of T-cell lineage, ie, CD4-T helper (1.17) and CD8-T suppressor (1.23) cells. Unexpectedly, strongly proliferating T-lymphoid leukemic cells were found to possess a plasma membrane characterized by a low FD value (1.10), close to the FD value measured on the plasma membrane of in vitro growing lymphoblasts derived from mature T-lymphocytes triggered by phytohemagglutinin (PHA), a mitogenic lectin. About $80 \%$ of acute leukemia subtypes of the B-cell lineage (c-ALL and pre-B undifferentiated phenotype) showed plasma membranes with FDs ranging from 1.12 to 1.17 , below the $\mathrm{FD}$ of the plasma membrane of differentiated B-lymphocytes. The remaining cases $(20 \%)$ of acute lymphoblastic B-leukemia showed a more convoluted cell surface with FD values of up to 1.24. The increase in FD, together with the accentuated coarseness of the nuclear surface, reflects significant changes in the DNA methylation pattern usually localized in heterochromatin nuclear regions and therefore was regarded as a bad prognostic factor for these patients. The usefulness of fractal analysis to assess the hematological cell phenotype and to define a clinical group was confirmed some 20 years later [22]. The analyse of "nuclei contours" of cells belonging to the B lineage, ie. normal lymphocytes and lymphoid cells isolated from patients with chronic lymphocytic leukemia (CLL), follicular 
lymphoma (FL), and diffuse large B-cell lymphoma (DLBCL) showed that the fractal dimension of perinuclear membranes were significantly different between the groups: reactive lymphocytes $(\mathrm{FD}=1.20)$ were situated between CLL $(\mathrm{FD}=1.25)$ and normal cells $(\mathrm{FD}=1.13)$, while aggressive lymphoma cells had a significantly higher fractal dimension ranging from 1.23 (FL) to 1.31 (DLBCL). By comparing data from the latter papers dealing with hematological malignancies it turned out that cells isolated from patients with different types of leukemia and/ or lymphoma have nuclear chromatin with roughness or complexity (high FD value) increasing with increasing degrees of aggressiveness and malignancy, whereas pericellular membranes acted inversely and looked smoother (low FD value) in cells having a high degree of malignancy. One could infer that hematological tumors did not undergo uniform neoplastic transformations, but rather manifest metabolic and phenotype changes that imply either an increasing or a decreasing complexity of the morphological surface and an altered organization of cell components mainly dependent upon the cytotype under investigation. This contrasts with the behavior of cell colonies of distinct breast cancer type and experimental tumors, which were observed to obey the same dynamics of proliferation and growth and display contours with self-similar fractal features when submitted to scaling analysis [23].

\section{Cancer tissues}

For an objective description of neoplastic and pathologic traits of cell tissues by the fractal approach, a main condition is the experimental definition of a scaling interval rather than a unique dimensional scale selected a priori. A critical reading of the literature shows that such a distinctive characteristic is insufficiently taken into account and inadequately applied in many investigations [24]. These views are typically interpreted in a qualitative manner by clinicians trained to classify abnormal features such as structural irregularities or high indices of mitosis. A more quantitative and hopefully more reproducible approach, which may serve as a useful adjunct to trained observers, is to analyze images with computational tools. Herein lies the potential of fractal analysis as a morphometric measure of the irregular structures typical of tumor growth. Among the most promising fields of investigation, for which fractal geometry provides an original approach and fractal dimension represents more than an additional geometrical parameter or just "a useful adjunct" are cell heterogeneity, architectural organization of tissues tumor, parenchymal border, cellular/nuclear morphology, developmental and morphogenetic processes in tissues and organs in healthy, pathologic, or tumor conditions, and the pathologies of the vascular architecture. Tumor grading on histology specimens is difficult to assess because tumors often consist of a heterogeneous mixture of cells with varying degrees of irregularity as well as local variations in cellular differentiation. Measuring the fractal dimension could aid pathologists in grading heterogeneity and in determining the spatial extent of poorly differentiated regions of breast and prostate tumors [25]. Cell heterogeneity, known to contribute in a determinant way to the histological grading of human breast cancer, has been examined by means of geostatistics and the Hurst fractal parameter [26]. Several examples seem to indicate that the occurrence of morphogenetic dynamics, the emergence of complex patterns, and the architectural organization of active tissues and tumor masses may be driven by constructive mechanisms related to fractal principles, including deterministic and/or random iteration of constituent units with varying degrees of selfsimilarity, scaling properties, and form conservation. Preservation of tissue architecture and cell polarity of organs and the eventual restoration of organized traits in tumor tissues, deconstructed and deregulated at various levels, is an emerging field of interest since it has been observed that biological entities organize with their own degrees of structural and behavioral complexity, develop on different spatial and time scales and follow modifications induced by drug or endocrine treatment as well $[18,27]$. It has been argued that reversion into a more "physiological" fractaldimension implies reduced morphologic instability and an increase of cells connectivity, what emphasizes the relevance of shape fractal parameters as descriptors of cell transition from one phenotype to another [15]. Fractal dimension has been used as a characterization parameter of premalignant and malignant epithelial lesions of the floor of the mouth in humans and of architectural changes of the epithelial connective tissue interface (ECTI) of the buccal mucosa during aging [28]. The onset of fundamental phenomena such as development, growth and cell death during different stages of carcinogenesis and cell differentiation, ie, from mesenchymal to smooth muscle cells, has been adequately investigated by fractal geometry as recently reported [29]. Fractal morphometry has provided quantitative information concerning the link between molecular, cellular and tissue changes during the development of canine tumors [30]. One highly promising approach appears to be a combination of fractal analysis, to provide a quantitative description of shapes, with radiographic imaging, which has the ability to discriminate malignant from benign tumor masses, and also from normal tissue structures [31]. The computed FD of the contour of a mass may be useful for characterizing shape and gray-scale complexity, which may vary between benign masses and malignant tumors in mammograms [32].

\section{The complexity of human brain and nervous cells}

The evolutionary concourse of two major events, "the tremendous expansion and the differentiation of the neocortex", has contributed to the development of the human brain [33]. Today, modern neurosciences recognize the presence of fractal properties in brain at various levels, i.e., anatomical, functional, pathological, molecular, and epigenetic [34], but not so long ago there was no analytical method able to objectively describe the complexity of biological systems such as the brain [35]. The intricacy of mammalian brain folds led Mandelbrot to argue that "A quantitative study of such folding is beyond standard geometry, but fits beautifully in fractal geometry". At that time however, there was no certainty about the brain's geometry or about neuron branching. Anatomical-histological evidence that the complexity of the plane-filling maze formed from dendrites of neural Purkinje cells of cerebellum was more reduced in non-mammalian species than in mammals led Mandelbrot to comment: "It would be very nice if this corresponded to a decrease in $\mathrm{D}$ (fractal dimension), but the notion that neurons are fractals remains conjectural" affirmed Mandelbrot. Since then, a wealth of investigations have documented the fractal organization of the brain and nervous tissue system, and implication of fractals for neurosciences has been unambiguously affirmed [36]. Among relevant applications of fractal analysis to nervous and brain tissue were pioneering studies which showed that the fractal dimension is an unbiased measure of the complexity of neuronal borders and branching pattern and of the time course of morphological development and differentiation of spinal cord neurons in culture, increasing from 1.1 for the less differentiated neuron up to 1.5 for the most differentiated cell [37-40]. Further studies have confirmed that the fractal dimension correlates with the increase in morphological complexity and neuronal maturity [41-45]. In some recent reports, rather exciting, it ha been argued that there is a trend towards a "Unified Fractal Model of the Brain" [46]. These authors 
suggested that the amount of information necessary to build just a tiny fraction of the human body, that is, just the cerebellum of the nervous system, was a task for which $1.3 \%$ of the information that the genome could contain was totally insufficient. "Fractal genome grows fractal organism; yielding the utility that fractality, e.g. self-similar repetitions of the genome can be used for statistical diagnosis, while the resulting fractality of growth, e.g. cancer, is probabilistically correlated with prognosis, up to cure" [47].

The brain is now accepted as one of nature's complete networks [48], while the hierarchical organization of the brain, seen at multiple scales from genes to molecular micronetworks and macronetworks organized in building neurons, has a fractal structure as well [49] with various modules that are interconnected in small-world topology [50]. The theoretical significance is that the fractality found in DNA and organisms, for a long time "apparently unrelated," was put into a "cause and effect" relationship by the principle of recursive genome function [47].

\section{References}

1. Mandelbrot B (1983) The Fractal Geometry of Nature. Freeman, San Francisco.

2. Leonardo da Vinci. Trattato della Pittura. ROMA MDCCCXVII. Nella Stamperia DE ROMANIS. A cura di Guglielmo Manzi Bibliotecario della Libreria Barberina.

3. Mandelbrot B (1977) Fractals, M.B. Form, Chance and Dimension. W.H. Freeman and Company, San Francisco.

4. Belaubre G (2006) L'irruption des Géométries Fractales dans les Sciences.Editions Académie Européenne Interdisciplinaire des Sciences (AEIS), Paris

5. Loud AV (1968) A quantitative stereological description of the ultrastructure of normal rat liver parenchymal cells. $J$ Cell Biol 37: 27-46. [Crossref]

6. Weibel ER, Stäubli W, Gnägi HR, Hess FA (1969) Correlated morphometric and biochemical studies on the liver cell. I. Morphometric model, stereologic methods, and normal morphometric data for rat liver. J Cell Biol 42: 68-91. [Crossref]

7. Mandelbrot B (1967) How long is the coast of britain? Statistical self-similarity and fractional dimension. Science 156: 636-638. [Crossref]

8. Paumgartner D, Losa G, Weibel ER (1981) Resolution effect on the stereological estimation of surface and volume and its interpretation in terms of fractal dimensions. J Microsc 121: 51-63. [Crossref]

9. Gehr P, Bachofen M, Weibel ER (1978) The normal human lung: ultrastructure and morphometric estimation of diffusion capacity. Respir Physiol 32: 121-140. [Crossref]

10. Rigaut JP (1984) An empirical formulation relating boundary length to resolution in specimens showing "non-ideally fractal" dimensions. J Microsc 13: 41-54.

11. Rigaut JP (1989) Fractals in Biological Image Analysis and Vision. In: Losa GA, Merlini D (Eds) Gli Oggetti Frattali in Astrofisica, Biologia, Fisica e Matematica, Edizioni Cerfim, Locarno, pp. 111-145.

12. Nonnenmacher TF, Baumann G, Barth A, Losa GA (1994) Digital image analysis of self-similar cell profiles. Int J Biomed Comput 37: 131-138. [Crossref]

13. Landini G, Rigaut JP (1997) A method for estimating the dimension of asymptotic fractal sets. Bioimaging 5: 65-70.

14. Dollinger JW, Metzler R, Nonnenmacher TF (1998) Bi-asymptotic fractals: fractals between lower and upper bounds. J Phys A Math Gen 31: 3839-3847.

15. Bizzarri M, Pasqualato A, Cucina A, Pasta V (2013) Physical forces and non linear dynamics mould fractal cell shape. Quantitative Morphological parameters and cell phenotype. Histol Histopathol 28: 155-174.

16. Losa GA, Nonnenmacher TF (1996) Self-similarity and fractal irregularity in pathologic tissues. Mod Pathol 9: 174-182. [Crossref]

17. Weibel ER (1991) Fractal geometry: a design principle for living organisms. $A m J$ Physiol 261: L361-369. [Crossref]

18. Losa GA (2012) Fractals in Biology and Medicine. In: Meyers R (Ed.), Encyclopedia of Molecular Cell Biology and Molecular Medicine, Wiley-VCH Verlag, Berlin.
19. Santoro R, Marinelli F, Turchetti G, et al. (2002) Fractal analysis of chromatin during apoptosis. In: Losa GA, Merlini D, Nonnenmacher TF, Weibel ER (Eds.), Fractals in Biology and Medicine. Basel, Switzerland. Birkhäuser Press 3: 220-225.

20. Bianciardi G, Miracco C, Santi MD et al. (2002) Fractal dimension of lymphocytic nuclear membrane in Mycosis fungoides and chronic dermatitis. In: Losa GA, Merlin D, Nonnenmacher TF, Weibel ER (Eds.), Fractals in Biology and Medicine. Basel, Switzerland, Birkhäuser Press.

21. Losa GA, Baumann G, Nonnenmacher TF (1992) Fractal dimension of pericellular membranes in human lymphocytes and lymphoblastic leukemia cells. Pathol Res Pract 188: 680-686. [Crossref]

22. Mashiah A, Wolach O, Sandbank J, Uzie IO, Raanani P, et al. (2008) Lymphoma and leukemia cells possess fractal dimensions that correlate with their interpretation in terms of fractal biological features. Acta Haematol 119: 142-150. [Crossref]

23. Brú A, Albertos S, Luis Subiza J, García-Asenjo JL, Brú I (2003) The universal dynamics of tumor growth. Biophys J 85: 2948-2961. [Crossref]

24. Baish JW, Jain RK (2000) Fractals and cancer. Cancer Res 60: 3683-3688.

25. Tambasco M, Magliocco AM (2008) Relationship between tumor grade and computed architectural complexity in breast cancer specimens. Hum Pathol 39: 740-746. [Crossref]

26. Sharifi-Salamatian V, Pesquet-Popescu B, Simony-Lafontaine J, Rigaut JP (2004) Index for spatial heterogeneity in breast cancer. J Microsc 216: 110-122. [Crossref]

27. Losa GA, Graber R, Baumann G, Nonnenmacher TF (1998) Steroid hormones modify nuclear heterochromatin structure and plasma membrane enzyme of MCF-7 Cells. A combined fractal, electron microscopical and enzymatic analysis. Eur J Histochem 42 : 1-9. [Crossref]

28. Landini G, Hirayama Y, Li TJ, Kitano M (2000) Increased fractal complexity of the epithelial-connective tissue interface in the tongue of 4 NQO-treated rats. Pathol Res Pract 196: 251-258. [Crossref]

29. Roy HK, Iversen P, Hart J, Liu Y, Koetsier JL, et al. (2004) Down-regulation of SNAIL suppresses MIN mouse tumorigenesis: modulation of apoptosis, proliferation, and fractal dimension. Mol Cancer Ther 3: 1159-1165. [Crossref]

30. Losa GA, De Vico G, Cataldi M (2009) Contribution of connective and epithelial tissue components to the morphologic organization of canine trichoblastoma. Connect Tissue Res 50: 28-29.

31. Li H, Giger ML, Olopade OI, Lan L (2007) Fractal analysis of mammographic parenchymal patterns in breast cancer risk assessment. Acad Radiol 14: 513-521. [Crossref]

32. Rangayyan RM, Nguyen TM (2007) Fractal analysis of contours of breast masses in mammograms. J Digit Imaging 20: 223-237. [Crossref]

33. De Felipe J (2011) The evolution of the brain, the human nature of cortical circuits, and intellectual creativity. Front Neuroanat 5: 29. [Crossref]

34. King RD, Brown B, Hwang M, Jeon T, George AT; Alzheimer's Disease Neuroimaging Initiative (2010) Fractal dimension analysis of the cortical ribbon in mild Alzheimer's disease. Neuroimage 53: 471-479. [Crossref]

35. Werner G (2010) Fractals in the nervous system: conceptual implications for theoretical neuroscience. Front Physiol 1: 15. [Crossref]

36. Losa GA (2014) On the Fractal Design in Human Brain and Nervous Tissue. Applied Mathematics 5: 1725-1732.

37. Smith TG Jr, Marks WB, Lange GD, Sheriff WH Jr, Neale EA (1989) A fractal analysis of cell images. J Neurosci Methods 27: 173-180. [Crossref]

38. Smith TG Jr, Bejar TN (1994) Comparative fractal analysis of cultured glia derived from optic nerve and brain demonstrated different rates of morphological differentiation. Brain Res 634: 181-190.

39. Smith TG Jr, Lange GD, Marks WB (1996) Fractal methods and results in cellular morphology--dimensions, lacunarity and multifractals. J Neurosci Methods 69: 123136. [Crossref]

40. Smith TG (1994) A Fractal Analysis of Morphological Differentiation of Spinal Cord Neurons in Cell Culture. In: Losa et al., (Eds.), Fractals in Biology and Medicine, Birkhäuser Press, Basel, vol.1.

41. Milosevic NT, Ristanovic D (2006) Fractality of dendritic arborization of spinal cord neurons. Neurosci Lett 396: 172-176. [Crossref]

42. Milosevic NT, Ristanovic D, Jelinek HF, Rajkovic K (2009) Quantitative analysis of 
dendritic morphology of the alpha and delta retinal ganglions cells in the rat: a cell classification study. J Theor Biol 259: 142-150. [Crossref]

43. Ristanovic D, Stefanovic BD, Milosevic NT, Grgurevic M, Stankovic JB (2006) Mathematical modelling and computational analysis of neuronal cell images: application to dendritic arborization of Golgi-impregnated neurons in dorsal horns of the rat spinal cord. Neurocomputing 69: 403-423.

44. Jelinek HF, Milosevic NT, Ristanovich D (2008) Fractal dimension as a tool for classification of rat retinal ganglion cells. Biol Forum 101: 146-150.

45. Bernard F, Bossu JL, Gaillard S (2001) Identification of living oligodendrocyte developmental stages by fractal analysis of cell morphology. J Neurosci Res 65: 439445. [Crossref]

46. Pellionisz A, Roy GR, Pellionisz PA, Perez JC (2013) Recursive genome function of the cerebellum: geometric unification of neuroscience and genomics. Berlin: In: Manto M, Gruol DL, Schmahmann JD, Koibuchi N and Rossi F (Eds.), Springer Verlag, "Handbook of the Cerebellum and Cerebellar Disorders". 1381-1423.

47. Pellionisz AJ (2008) The principle of recursive genome function. Cerebellum 7: 348 359. [Crossref]

48. Di Ieva A, Grizzi F, Jelinek H, Pellionisz AJ, Losa GA (2015) Fractals in the Neurosciences, Part I: General Principles and Basic Neurosciences. The Neuroscientist $\mathrm{XX}(\mathrm{X}) 1-15$.

49. Pellionisz A (1989) Neural geometry: towards a fractal model of neurons. Cambridge: Cambridge University Press.

50. Agnati LF, Guidolin D, Carone C, Dam M, Genedani S, et al. (2008) Understanding neuronal molecular networks builds on neuronal cellular network architecture. Brain Res Rev 58: 379-99. [Crossref]

Copyright: (C2015 Losa GA. This is an open-access article distributed under the terms of the Creative Commons Attribution License, which permits unrestricted use, distribution, and reproduction in any medium, provided the original author and source are credited. 